\title{
Nonlinear Vibration Analysis for a Simply Supported Beam Elastically Colliding with Clamped Springs at Arbitrary Locations
}

\author{
Tatsuhito Aihara
}

\begin{abstract}
This paper deals with the analysis of collision vibration in continuous system excited by periodic displacement with arbitrary function. The analytical model is steady collision vibration of simply supported beam which collides with arbitrary location. In order to clarify the main resonance of the system, the resulting vibration is analyzed applying the Fourier series method for this system and an exact solution is proposed. Following the theoretical analysis, numerical calculation is performed and experiments are also carried out to verify the theoretical results.
\end{abstract}

Index Terms - Vibration analysis, theory of vibration, forced vibration, collision vibration.

\section{INTRODUCTION}

Collision vibrations are generated by a high-speed collision with an elastic body and constitute a system with a comparatively high degree of nonlinearity making it difficult to perform quantitative assessment to obtain general analytical results. Choi [1] analyzed the steady forced collision response of a single-degree-of-freedom system with an asymmetrical piecewise linear restoring force. The restoring force was derived using a fast Fourier transformation algorithm, and the method of harmonic balance was applied. The nonlinear simultaneous equations derived by harmonic balance were solved by using a Newton-Raphson iteration method, and periodic solutions were calculated. Studies on collision vibrations of continuous systems include analyses by Watanabe [2], Shaw [3], Aoki [4] and Brake [5] of the responses under single harmonic excitation. However, seismic design of chemical plant structures such as piping systems must consider not only single periodic excitation. It must also consider a wide variety of exciting vibrations of structures [6]. Kumano [7] have also examined analyzed the steady forced collision response of a continuous system having a symmetrical piecewise linear restoring force by the Fourier series method. The main resonance was analyzed and an exact solution was derived. Numerical calculations were also performed based on the exact solution and resonance curves were constructed. The effect of the nonlinearity on the resonance was shown. These continuous system analyses were limited to special cases in terms of the locations of elastic collisions, with collisions occurring at the free end of a cantilever beam or at

\footnotetext{
Manuscript received May 11, 2015; revised October 5, 2015.

Tatsuhito Aihara is with the Department of Mechanical Engineering, Hosei University, Tokyo 184-8584, Japan (e-mail: tatsuhito.aihara@hosei.ac.jp).
}

the midpoint of a simply supported beam at both ends. A real structure or machine often contains clearance or gaps mid-span, as well as regions where structural elements or components undergo elastic collision mid-span with other components. However, no analyses have been appeared to be conducted for periodic excitations with arbitrary functions based on displacement for these structural materials.

This paper presents basic research on analyzing the steady collision vibrations arising in a simply supported beam suspended between two springs clamped symmetrically at an arbitrary position along the beam. This study used Fourier series method, which expands a series about the restoring force that arises from a collision, and a rigorous expression for the resulting vibration was derived. A numerical calculation was also performed based on the analytical results. An experiment was also conducted with physical conditions matching those used in the simulation; the experimental results were in good agreement with the numerical predictions, confirming the effectiveness of this analysis.

\section{THEORETICAL ANALYSIS}

\section{A. Characteristics of System and Equation of Motion}

The system examined in this study is shown in Fig. 1. It is a simply supported beam subjected to elastic collision at an arbitrary point along its span with springs clamped to walls located at symmetric distances from the neutral beam position, while subjected to a displacement of a periodic excitation with an arbitrary function $q(t)$.

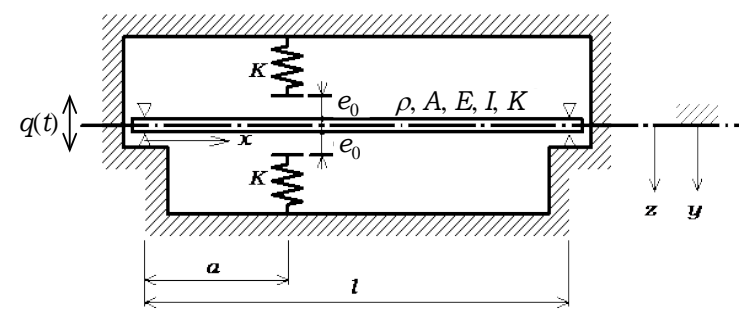

Fig. 1. Dynamical model of collision vibration system.

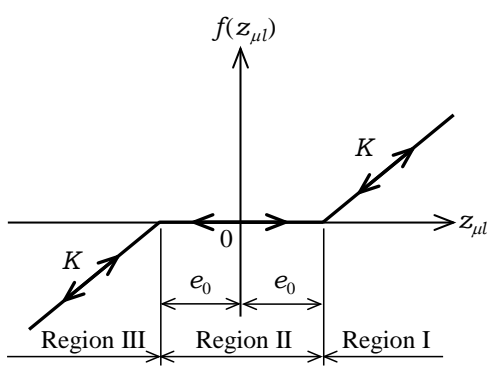

Fig. 2. Characteristics of restoring force. 
As shown in Fig. 2, the restoring force of the spring is symmetric piecewise linear with a dead zone (zone II) at clearance $z=\left[+e_{0},-e_{0}\right]$. If zone II is considered the standard, then zones I and III as an entire system can be considered the nonlinear regions. If $A$ is the cross section of the beam, $I$ is the second moment of the beam cross-sectional area, $E$ is Young's modulus and $\rho$ is the density, we can calculate the transverse vibration in bending under periodic excitation by a displacement described by an arbitrary function $q(t)$. During this vibration, the beam also collides elastically with a spring with a spring constant of $K$ at an arbitrary position along the beam. We consider the beam to consist of two independent bodies on either side of this location: beam I on the side with the left end and beam II on the side with the right end. The relative displacement of beam $i$ (where $i=\mathrm{I}, \mathrm{II}$ ) is $z_{i}$. The following equation of motion is obtained for $z_{i}$ :

$$
\frac{\partial^{2} z_{i}}{\partial t^{2}}+\frac{E I}{\rho A} \cdot \frac{\partial^{4} z_{i}}{\partial x^{4}}=-\frac{\partial^{2}}{\partial t^{2}} q(t), \quad(i=\mathrm{I}, \mathrm{II})
$$

The displacement excitation $q(t)$ acting on the system is assumed to be described by an arbitrary periodic function, and can therefore be expanded into the following complex Fourier series and the real Fourier series given later.

$$
q(t)=\sum_{n=-\infty}^{\infty} s_{n} e^{j n \omega t}=\frac{f_{0}}{2}+\sum_{n=1}^{\infty}\left(f_{n} \cos n \omega t+g_{n} \sin n \omega t\right)
$$

where $\omega$ is the angular frequency and $S_{n}, f_{0}, f_{n}$ and $g_{n}$ are Fourier coefficients. If (2) is substituted into (1), we obtain the following partial differential equation:

$$
\frac{\partial^{2} z_{i}}{\partial t^{2}}+\frac{E I}{\rho A} \frac{\partial^{4} z_{i}}{\partial x^{4}}=\sum_{n=-\infty}^{\infty} s_{n} n^{2} \omega^{2} e^{j n \omega t}
$$

We consider the steady collision vibration in this analysis, so the relative beam displacement $z_{i}$ is a periodic function of the angular frequency $\omega$ and $q(t)$. It follows that the resulting displacement $z_{i}$ in (3) can be expanded into the following Fourier series, in which the superscript $i$ denotes beam I or II:

$$
Z_{i}=\sum_{n=-\infty}^{\infty} W_{n}^{i}(x) e^{j n \omega t}
$$

The resulting displacement (4) can be substituted into the partial differential equation in (3). If we use the relation between the relative displacement and the absolute displacement, we obtain the following ordinary differential equation for the beam:

$$
\frac{d^{4} X_{n}^{i}(x)}{d x^{4}}=\frac{\rho A}{E I} n^{2} \omega^{2} X_{n}^{i}(x)
$$

where $X_{n}^{i}(x)$ ( $\left.i=\mathrm{I}, \mathrm{II} ; n=0,1,2, \ldots\right)$ is an eigenfunction describing normal-mode vibration. If the coefficients in (5) are then restated as

$$
\lambda_{n}^{4}=n^{2} \omega^{2} \frac{\rho A}{E I}
$$

then $\lambda_{n}$ are the eigenvalues and $X_{n}^{i}(x)$ are given by the following formula:

$$
\left.\begin{array}{l}
X_{n}^{\mathrm{I}}(x)=A_{n} \cos \lambda_{n} x+B_{n} \sin \lambda_{n} x+C_{n} \cosh \lambda_{n} x+D_{n} \sinh \lambda_{n} x \\
X_{n}^{\mathrm{II}}(x)=E_{n} \cos \lambda_{n} x+F_{n} \sin \lambda_{n} x+G_{n} \cosh \lambda_{n} x+H_{n} \sinh \lambda_{n} x
\end{array}\right\}
$$

The $A_{n}, B_{n}, C_{n}, \ldots H_{n}$ in the above equation are constants to be determined later from the boundary conditions and from the continuity conditions. The primary angular frequency of a cantilever beam is given by

$$
\omega_{1}=\frac{Z_{1}^{2}}{l^{2}} \sqrt{\frac{E I}{\rho A}}
$$

where $Z_{1}=\pi$. Equation (6) can be restated in terms of the frequency ratio $\Omega=\omega / \omega_{1}$, giving the following relationship:

$$
\lambda_{n}^{4} l^{4}=Z_{1}^{4} n^{2} \Omega^{2}
$$

\section{B. Fourier Series Method}

This analysis limits considerations to resulting waves in which the beam strikes and compresses the upper and lower springs in the nonlinear region only one time each during the main resonance region. In order to simplify the analysis, the response waveform was assumed to be approximately axially symmetric about the midpoint of the duration of beam's collision with each of the clamped springs and the maximum or minimum resulting displacement is assumed to occur at those temporal midpoints. One cycle of the resulting wave of the relative displacement at the location of collision with the springs is divided into three zones (I, II, III). These zones correspond to zones I, II and III in the restoring force characteristic graph in Fig. 2. The phase angle $\theta$ is introduced here in terms of the portion of the cycle $\theta_{0}$ spent in contact with one or the other spring. The starting point for the period is considered to be the midpoint of zone I. The phase lag angle $\alpha$ is defined as

$$
\theta=\omega t-\alpha
$$

$\alpha$ is unknown for now and will be calculated later, and $\omega$ is the angular frequency of excitation.

The boundary conditions for the analytical model shown in Fig. 1 are set using the following expressions:

$$
\begin{aligned}
& x=0: z_{\mathrm{I}}=0, \quad x=0: \frac{d^{2} z_{\mathrm{I}}}{d x^{2}}=0 \\
& x=l: z_{\text {II }}=0, \quad x=l: \frac{d^{2} z_{\text {II }}}{d x^{2}}=0
\end{aligned}
$$

The dwell angle $\theta_{0}$ is defined as the portion of the resulting vibration period spent in nonlinear zones I and III. The following expressions show the conditions at the collision point when the beam enters and leaves the nonlinear regions (the switching-over conditions):

$$
\theta= \pm \frac{\theta_{0}}{2} \quad: z_{\mu l}=e_{0}, \quad \theta=\pi \pm \frac{\theta_{0}}{2} \quad: z_{\mu l}=-e_{0}
$$

where $\mu(=a / l)$ is collision positon ratio and $z_{\mu l}$ express collision point.

There is continuity at the boundary point between beam I and beam II. The continuity conditions are as follows:

$$
\begin{aligned}
& z_{\text {I }}=z_{\text {II }}, \quad \frac{d z_{\text {II }}}{d x}=\frac{d z_{\text {II }}}{d x}, \\
& \frac{d^{2} z_{\text {I }}}{d x^{2}}=\frac{d^{2} z_{\text {II }}}{d x^{2}}, \quad E I\left(\frac{d^{3} z_{\text {I }}}{d x^{3}}-\frac{d^{3} z_{\text {II }}}{d x^{3}}\right)=f\left(z_{\mu l}\right)
\end{aligned}
$$


In (13), $f\left(z_{\mu l}\right)$ is the restoring force in the spring during elastic collision with the beam, as shown in Fig. 2; this is written $g(\theta) . \theta_{0}$ is the dwell angle, the angle of duration of collision points between the beam and spring in zones I and III. Thus, $g(\theta)$ is found as follows:

$$
\left.\begin{array}{l}
g(\theta)=K\left(z_{\mu l}-e_{0}\right):-\frac{\theta_{0}}{2} \leq \theta \leq \frac{\theta_{0}}{2} \\
g(\theta)=0 \quad: \frac{\theta_{0}}{2} \leq \theta \leq \pi-\frac{\theta_{0}}{2}, \pi+\frac{\theta_{0}}{2} \leq \theta \leq 2 \pi-\frac{\theta_{0}}{2} \\
g(\theta)=K\left(z_{\mu l}+e_{0}\right): \pi-\frac{\theta_{0}}{2} \leq \theta \leq \pi+\frac{\theta_{0}}{2}
\end{array}\right\}
$$

$g(\theta)$ can be considered a periodic function of the phase angle $\theta$ with period $2 \pi$. Thus, it can be expanded into a Fourier series:

$$
g(\theta)=\sum_{n=-\infty}^{\infty} c_{n} e^{j n \theta}=\frac{a_{0}}{2}+\sum_{n=1}^{\infty}\left(a_{n} \cos n \theta+b_{n} \sin n \theta\right)
$$

where $c_{n}, a_{0}, a_{n}$ and $b_{n}$ are Fourier coefficients.

\section{Derivation of Analytical Equation}

The dimensionless resulting displacement ratio $z_{\mu l} / e_{0}$ was derived as follows by using (3) and (13):

$$
\frac{z_{\mu 1}}{e_{0}}=\frac{\cos \theta+\sum_{n=3,5,7, \ldots}^{\infty}\left\{\left(N_{n} v_{n} \frac{1-M_{1} x_{1}}{N_{1}}+M_{n} y_{n}\right)\left(\sin n \theta-\frac{1-M_{1} x_{1}}{N_{1}}+M_{n} x_{n}\right)\left(\sin \frac{n \theta_{0}}{2} \sin \frac{\theta_{0}}{2} \sin \theta\right)\right\}}{\cos \frac{n \theta_{0}}{2}+\sum_{n=3,5,7, \ldots}^{\infty}\left(N_{n} u_{n} \frac{1-M_{1} x_{1}}{N_{1}}+M_{n} x_{n}\right) \cos \frac{n \theta_{0}}{2}}
$$

The following values are derived for the excitation amplitude ratio $f_{1} / e_{0}$ and the phase lag angle $\alpha$ corresponding to these conditions:

$$
\begin{aligned}
& \frac{f_{1}}{e_{0}}=\frac{1}{\cos \alpha} \frac{1-M_{1} x_{1}}{N_{1}} / \sum_{n=1,3,5, \cdots}^{\infty}\left(N_{n} u_{n} \frac{1-M_{1} x_{1}}{N_{1}}+M_{n} x_{n}\right) \cos \frac{n \theta_{0}}{2} \\
& \alpha=\tan ^{-1} \frac{\left\{M_{1} y_{1}+\sum_{n=3,5,7, \ldots}^{\infty}\left(N_{n} v_{n} \frac{1-M_{1} x_{1}}{N_{1}}+M_{n} y_{n}\right) \frac{\sin \frac{n \theta_{0}}{2}}{\sin \frac{\theta_{0}}{2}}\right\}}{\left(1-M_{1} x_{1}\right)}
\end{aligned}
$$

These dimensionless equations were introduced as simplified forms of (16)-(18):

$$
x_{n}=\frac{a_{n}}{k \Gamma}, \quad y_{n}=\frac{b_{n}}{k \Gamma}
$$

Equations (16)-(18) can all be determined using the three parameters of frequency ratio $\Omega$, phase lag angle $\alpha$ and dwell phase angle $\theta_{0}$ in zones I and III and the independent dimensionless Fourier coefficients $x_{n}$ and $y_{n}$. Therefore, once $x_{n}$ and $y_{n}(n=1,3,5, \ldots)$ are known, the resulting displacement ratio $z_{\mu l} / e_{0}$ can be found from (16), the corresponding excitation amplitude ratio $f_{1} / e_{0}$ can be found from (17), and the phase lag angle $\alpha$ can be found from (18).

\section{Identification of the Dimensionless Fourier Coefficients}

The first and second expressions in (19) define the dimensionless Fourier coefficients $x_{n}$ and $y_{n}$. The requirement to satisfy the piecewise linear condition of the restoring force provides the following infinite set of simultaneous linear equations:

$$
\begin{aligned}
& x_{m}-\sum_{n=1,3,5, \cdots}^{\infty}\left(A_{m n} x_{n}-B_{m n} x_{1}\right)=\sum_{n=1,3,5, \cdots}^{\infty} I_{m n}(m=1,3,5 \cdots) \\
& y_{m}-\sum_{n=1,3,5, \cdots}^{\infty}\left(C_{m n} y_{n}-D_{m n} x_{1}\right)=\sum_{n=1,3,5, \cdots}^{\infty} J_{m n}(m=1,3,5 \cdots)
\end{aligned}
$$

Thus, solving (20) and (21) together determines $x_{n}$ and $y_{n}$. $A_{m n}, B_{m n}, \ldots J_{m n}$ in the above are constants determined by $\Omega$, $\alpha$, and $\theta_{0}$.

\section{NUMERICAL AND EXPERIMENTAL RESULTS}

\section{A. Comparison of Numerical Results with Experimental} Results

Fig. 3 shows the resonance curve that compares the numerically predicted amplitudes at resonance at the collision position during unitary excitation and the observed amplitudes in the experiment. These results agree reasonably well. The resonance curve bends to the right when the maximum vibration amplitude at the collision point entered the nonlinear region (marked with $\bullet$ ), beyond which the curve bent to the right, indicating prominent nonlinear behavior.

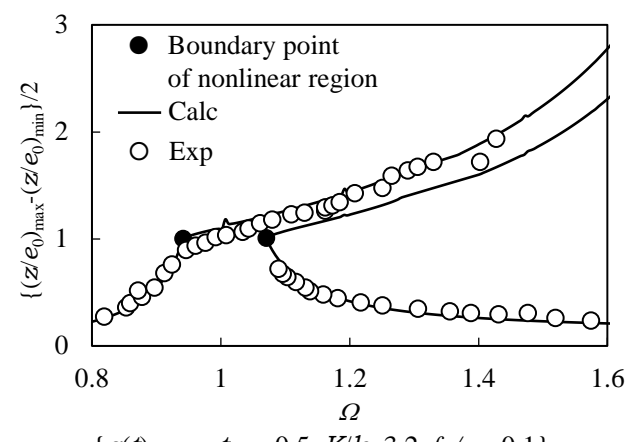

$\left\{q(t)=\cos \omega t, \mu=0.5, K / k=3.2, f_{1} / e_{0}=0.1\right\}$

Fig. 3. Resonance curve for comparing theoretical result with experimental one.

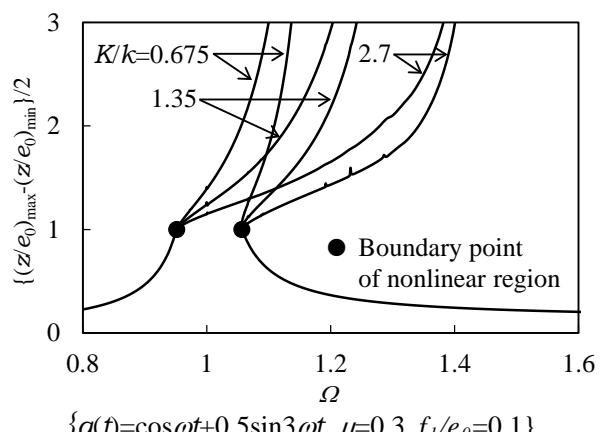

Fig. 4. Resonance curves in the case where spring constant ratio $K / k$ is parameter.

\section{B. Example of Numerical Calculation}

Numerical calculations were performed using the results described above and a periodic solution was found for steady vibration in the main resonance regions. A periodic excitation with an arbitrary function was assumed.

Fig. 4 shows the resonance curve when the spring constant 
ratio $K / k$ is parameter. $K / k$ expresses the magnitude of the nonlinearity of the supporting spring; when the resonance curve enters the nonlinear region, it bends, and the greater $K / k$ is, the more the resonance curve bends to the right.

Fig. 5 shows the resonance curve with respect to another parameter $f_{1} / e_{0}$, the ratio of excitation amplitude. The greater $f_{1} / e_{0}$ is, the wider the extent of the resonance region, and the lower $e_{0}$, the relative width of the linear region (Region I) is, the wider the resonance region.

Fig. 6 shows the resonance curve when the collision position ratio $\mu$ is parameter. Fig. 6 show that the greater $\mu$ is, the more the resonance curve bends to the right.

\section{CONCLUSIONS}

This study examined the vibration of a simply supported beam elastically colliding with springs at an arbitrary location along the span of the beam as an example of the behavior of a continuous system responding to a vibratory excitation. The following results were obtained:

1) The analyzed model consisted of a cantilever beam elastically colliding with clamped springs on both sides, placed at arbitrary locations along the span of the beam. There were symmetric gaps between the rest position of the beam and each spring, leaving a dead zone in the symmetric piecewise linear system. Fourier series method was employed to examine the steady collision vibration resulting from a periodic excitation with an arbitrary function. A rigorous solution was found for the resulting vibration.

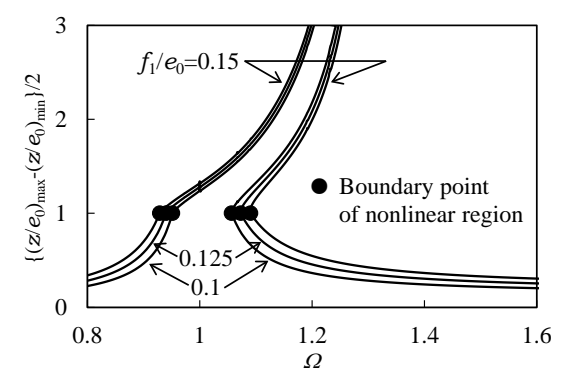

$\{q(t)=\cos \omega t+0.5 \sin 3 \omega t, \mu=0.3, K / k=1.35\}$

Fig. 5. Resonance curves in the case where excitation amplitude ratio $f_{1} / e_{0}$ is parameter.

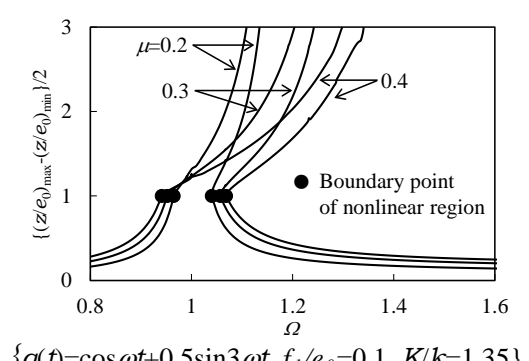

$\left\{q(t)=\cos \omega t+0.5 \sin 3 \omega t, f_{1} / e_{0}=0.1, K / k=1.35\right\}$

Fig. 6. Resonance curves in the case where collision position ratio $\mu$ is parameter.

2) A physical model was constructed and subjected to a cosine excitation as an example of periodic excitation with an arbitrary function to verify the results of the analysis conducted in (1). The experimental results were compared with the analytical results and found to agree with them well.

3) A numerical model was executed on the basis of the analysis in (1). Parameters were created of the excitation amplitude ratio $f_{1} / e_{0}$, spring collision position ratio $\mu$ and nonlinearity (ratio of spring constants) $K / k$ to produce graphs of resonance curves and the effect of these factors on the resonance curve shape were identified numerically.

4) It is possible to treat periodic excitations with an arbitrary function in a general manner. Once this analytical method is firmly established, it will be possible to use the procedure in (3) to calculate solutions for resulting vibrations for any magnitude of the parameters in (3).

\section{REFERENCES}

[1] Y. S. Choi and S. T. Noah, "Periodic forced vibration of unsymmetrical piecewise-linear systems by incremental harmonic balance method," Journal of Sound and Vibration, vol. 121, no. 1, pp. 117-126, 1988.

[2] T. Watanabe, "Forced vibration of continuous system with nonlinear boundary conditions," Transactions of the ASME, Journal of Mechanical Design, vol. 100, pp. 487-491, 1978.

[3] S. W. Shaw, "Forced vibrations of a beam with one-sided amplitude constraint: theory and experiment," Journal of Sound and Vibration, vol. 99, no. 2, pp. 199-212, 1985.

[4] S. Aoki and Y. Sakai, "Analysis of stationary impact vibration in continuous system with asymmetrical hysteresis loop characteristics," Transactions of the Japan Society of Mechanical Engineers, vol.76, no.767, pp. 1692-1699, 2010 (in Japanese).

[5] M. R. Brake, "A hybrid approach for the modal analysis of continuous systems with discrete piecewise-linear constraints,” Journal of Sound and Vibration, vol. 330, no. 13, pp. 3196-3221, 2011.

[6] T. Watanabe, K. Suzuki, T. Mitsumori, and N. Shimizu, "Seismic responses of plant piping system having friction and vibro-impact characteristics," Transactions of the Japan Society of Mechanical Engineers, vol.62, no.595, pp. 873-878, 1996 (in Japanese).

[7] H. Kumano, N. Nagao, and K. Temma, “Analysis of steady impact vibration in continuous system excited by periodic displacement with arbitrary function (The case where analytical model is simply-supported-beam with an attached mass)," Transactions of the Japan Society of Mechanical Engineers, vol. 71, no. 712, pp. 3367-3374, 2005 (in Japanese)

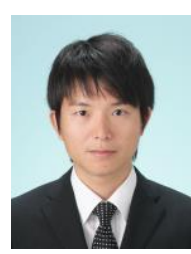

Tatsuhito Aihara received his $\mathrm{PhD}$ in engineering from Tokyo Metropolitan University, Japan in 2013.

From 2009 to 2014, he was a researcher with Toyota Central R\&D Labs. Currently, he is an associate professor at the Department of Mechanical Engineering, Hosei University, Japan. His research interests are in theoretical analysis for nonlinear vibrations, multi body dynamics and acoustics analysis. He is a member of JSME, JSDE and ASJ. 